\title{
Comparative study of spirometric parameters between active tobacco smokers and tobacco non-smokers
}

\author{
Dr. Ritesh M. Karia ${ }^{1}$, Dr. Pradnya A. Gokhale ${ }^{2}$, Dr. Hemant B. Mehta ${ }^{3}$ \\ ${ }^{1}$ Assistant Professor, Department of Physiology, GMERS Medical College, Dharpur-Patan, Gujarat, India, \\ ${ }^{2}$ Additional Professor, Department of Physiology, Govt. Medical College, Bhavnagar, Gujarat, India \\ ${ }^{3}$ Prof \& Head, Department of Physiology, Govt. Medical College, Bhavnagar, Gujarat, India
}

\begin{abstract}
:
Aim of this study is to study effect of smoking on various spirometric parameters like forced vital capacity (FVC), forced expiratory volume in 1 second $\left(\mathrm{FEV}_{1}\right)$ and $\mathrm{FEV}_{1} / \mathrm{FVC}$ ratio in apparently healthy tobacco smokers and nonsmokers and to compare the result of both the studies to assess the effects of smoking. The present study was carried out by computerized software of Pulmonary Function Test named 'Spiro Excel' on 50 non-smokers and 50 smokers. Smokers are divided in three gropus. Full series of test take 4 to 5 minutes. Tests were compared in the both smokers and non-smokers group by the 'unpaired t test'. Statistical significance was indicated by ' $p$ ' value $<0.05$. From the result it is found that actual value of spirometric parameters are significantly lower in all smokers group than non-smokers.
\end{abstract}

Key Words: Effect of smoking, forced vital capacity, forced expiratory flow rate in 1 second, $\mathrm{FEV}_{1} / \mathrm{FVC}$ ratio

\section{Introduction}

Spirometery is a test to examine functional capacity of lungs and respiratory system. The most common parameters measured are Forced vital capacity $(\mathrm{FVC})$, Forced expiratory volume in 1 second $\left(\mathrm{FEV}_{1}\right)$ and $\mathrm{FEV}_{1} / \mathrm{FVC}$ ratio. It is estimated that nearly $50 \%$ of men are dependent on some form of tobacco use ${ }^{1}$. The overall prevalence of current tobacco use from The National Household Survey of Drug and Alcohol Abuse in India( NHSDAA) was 55.8\% ${ }^{2}$. Tobacco smoke is mixture of more than 4000 compunds $^{3}$. Out of these many compounds are known to be carcinogenic and toxic. It can cause various pathophysiological effects. It affect the respiratory system first. It causes changing in the central and peripheral airways, alveoli and capillaries. It affects all the parameter of Pulmonary Function Test.

\section{Material and Method:}

The present study was carried out at Pulmonary Function Lab, department of Physiology, Govt. Medical College, Bhavnagar, Gjarat, India in 100 male subjects. Out of them 50 were non-smokers and 50 were smokers. Smokers are divided in three gropus ${ }^{4}$ :

Group 1 : Mild Smokers -A person who smokes 10 or less than 10 tobacco products per day.

Group 2 : Moderate Smokers - A person who smokes more than 10 but less than 20 tobacco products per day.

Group 3 : Heavy Smokers - A person who smokes more than 20 tobacco products per day.

The control group (non-smokers) was comparable in age, sex, economic status, socio-physical activity to study group

The present study was carried out by computerized software of Pulmonary Function Test named 'SPIRO EXCEL'. Spiro Excel is an instrument designed for lung function screening; the core of the system is the 'intelligent' flow meter that, connected through the USB cable, turns any personal computer (laptop or desktop) in a complete spirometric lab.

Spiro Excel is designed in such a way that it is easy and simple to operate and give highly accurate results. With the help of Spiro Excel it is easy to analyze data and it gives accurate result without manual calculation according to standardize testing protocol and predictions.

\subsection{Subject Preparation:}

All the subject were physically healthy, without any symptoms. The experimental protocol was explained to all the subjects and written consent was obtained from them. Subject was explained and demonstrated about the procedure to be performed. All anthropometric measurement (age, height and weight) were obtained in the subjects wearing light-weight clothing and barefoot and at room temperature. All vital Data (temperature, pulse, respiratory rate and blood pressure) was collected in sitting position after making subject relax and calm ${ }^{5}$. General Examination and Systemic Examination 
(complete RS and CVS examination) was done according to standard protocol. They were allowed to do enough practice, as lung volume depends on the subject's making a maximal voluntary effort. Full series of test take 4 to 5 minutes.

Following activities was avoided prior to test which was approved by American Thoracic Society(ATS ${ }^{6}$.

- $\quad$ Smoking within at least $1 \mathrm{hr}$ of testing

- $\quad$ Consuming alcohol within $4 \mathrm{hr}$ of testing

- Performing vigorous exercise within 30 min of testing

- Wearing clothing that substantially restricts full chest and abdominal expansion

- $\quad$ Eating a large meal within $2 \mathrm{hr}$ of testing.

\subsection{Statical Analysis:}

The value of $\mathrm{FVC}, \mathrm{FEV}_{1}$ and $\mathrm{FEV}_{1} / \mathrm{FVC}$ were compared in the both smokers and non-smokers group by the 'unpaired $t$ test'. Data were expressed in mean \pm SD. Statistical significance was indicated by ' $p$ ' value $<0.05$.

\section{Results:}

Actual value and predicted value of $\mathrm{FVC}, \mathrm{FEV}_{1}$ and $\mathrm{FEV}_{1} / \mathrm{FVC}$ are shown in Figure 1, 2 and 3.

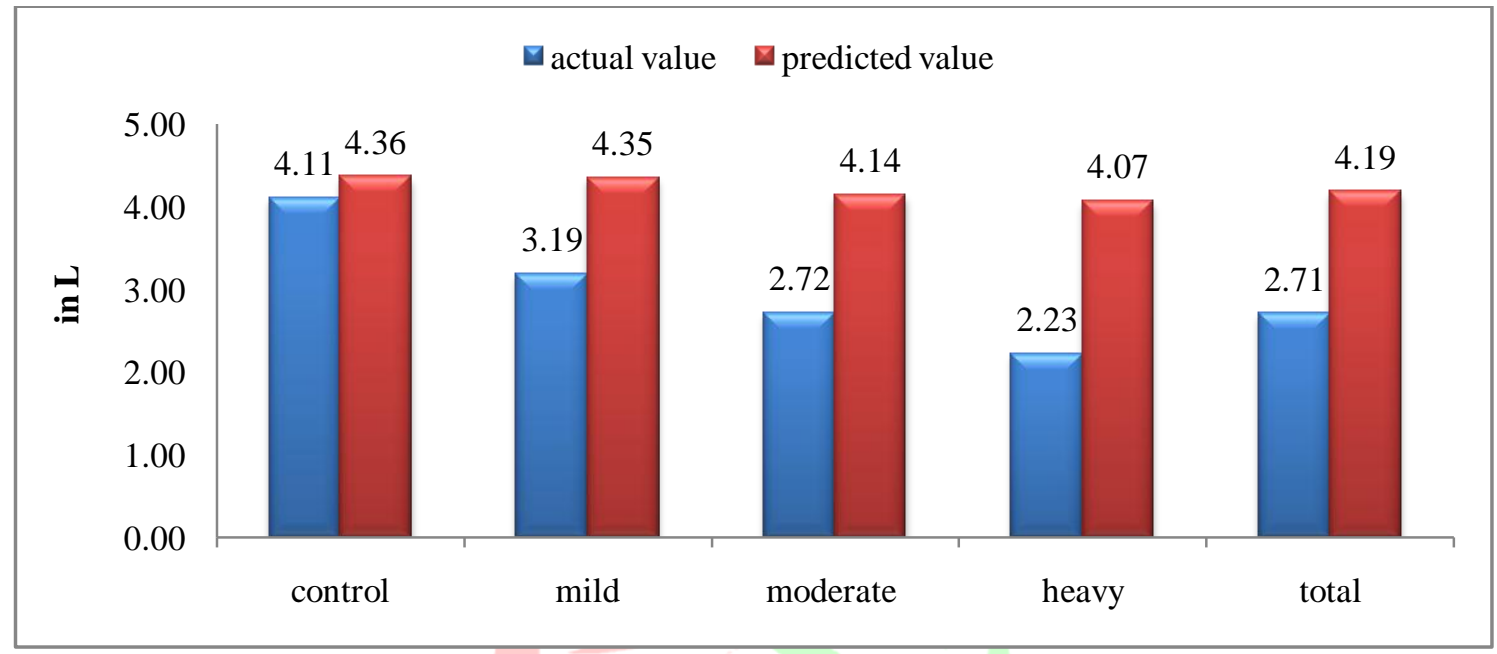

Fig. 1: shows comparison between actual mean value and predicted mean value of FVC

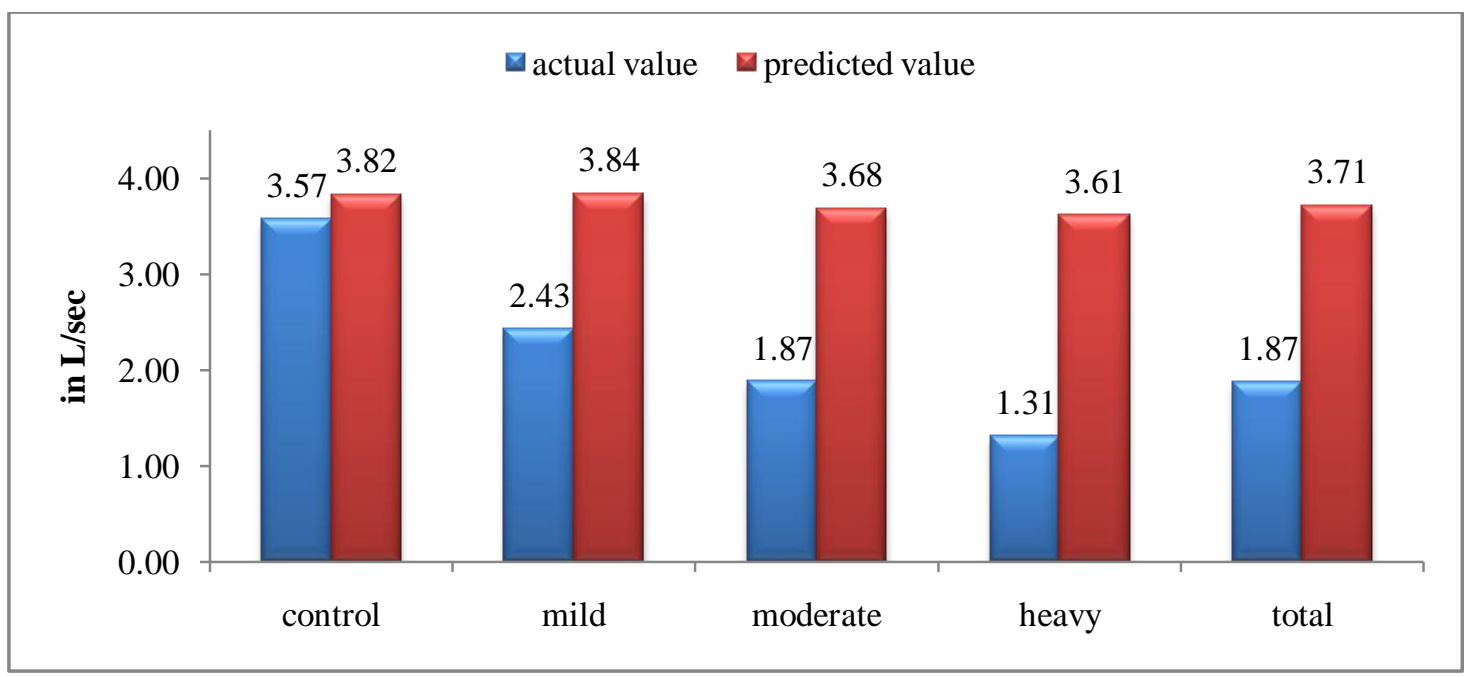

Fig. 2: shows comparison between actual mean value and predicted mean value of $F E V_{1}$ 


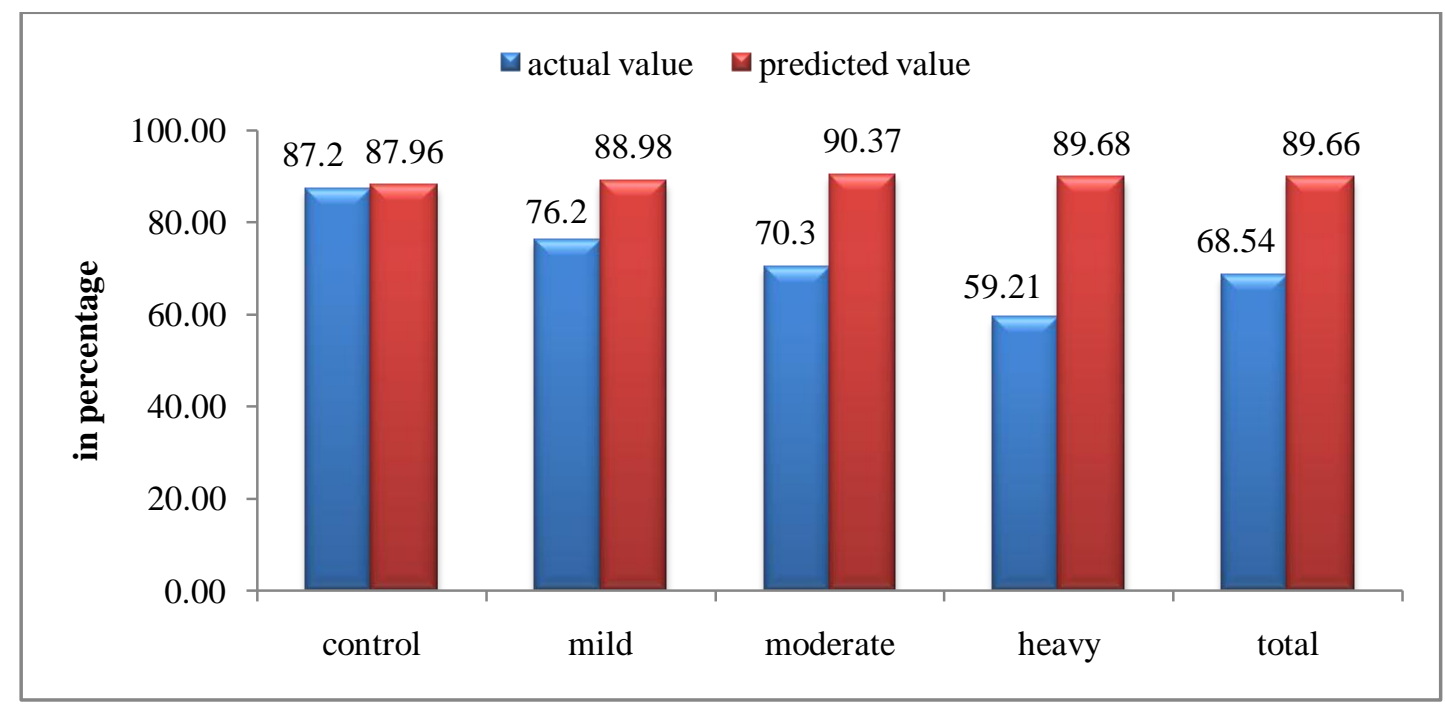

Fig. 3: shows comparison between actual mean value and predicted mean value of $F E V_{1} / F V C$

Non-smoker group shows non-significant change in spirometric parameters than their predicted value. Smokers groups shows significantly lower value than their predicted value. The difference of actual value and predicted value of spirometric parameters increase as the degree of smoking increases.

\section{Conclusion:}

Therefore, it is concluded that value of spirometric parameters are lower in active tobacco smokers than tobacco non-smokers. The actual value of spirometric parameters decrease as the number of tobacco smoking products increase. Therefore pulmonary function is lower in moderate smokers than in mild smokers and lower in heavy smokers than in mild and moderate smokers. Lower pulmonary function are associated with greater risk for lung disease, cardiovascular disease, cancer and other disease.

So, aggressive tobacco control programme aimed to inform the public about the hazards of tobacco use and to provide restriction on the use of or purchase of tobacco must be started. This will be helpful to change policies towards tobacco use, in order to prevent tobacco induced morbidity and mortality.

\section{Acknowledgment:}

We are thankful to subjecst who give us permission to study on them and made this study possible.

\section{References:}

[1] Ministry World Health Organization, Tobacco or Health : A Global status Organization, 1997, 414-17

[2] Report on Tobacco Control in India (New Delhi, India), 25 November 2004 of Health \& Family Welfare, Nirman Bhawan, Maulana Azad Road, New Delhi 110011, India

[3] Newcomb PA. The health consequences of smoking cancer : cigarette smoking. Med CI North Am 76, 1992 , 305-31

[4] R.W.Colin. Clinical Findings, Sputum Examinations, and Pulmonary Function Tests Related to smoking Habit of 500 women. Chest: 66, 1974, 652-659.

[5] Brusasco, R. Crapo and G. Viegi. Series ATS/ERS Task Force: Standardisation Of Lung Function Testing. Eur Respir J, 262005 153-161

[6] Joshi A,. Correclation of Pulmonary Function Tests with Body Fat Percentage in young Individuals. Indian J Physio Pharmacol 52 (4), 2008; 383-388. 